\title{
Coronal Density Measurements Using Giant Radio Pulses of the Crab Pulsar at the Cycle 24/25 Minimum
}

\author{
Munetoshi Tokumaru ${ }^{1}$ (D) Ryuya Maeda ${ }^{1,2} \cdot$ Kaito Tawara $^{1,3} \cdot$ Kazuhiro Takefuji $^{4}$. \\ Toshio Terasawa ${ }^{5}$
}

Received: 17 September 2021 / Accepted: 16 December 2021 / Published online: 17 January 2022

(c) The Author(s) 2022

\begin{abstract}
Accurate measurements of the coronal plasma density profile, which varies with the solar cycle (SC), are necessary to elucidate the solar wind acceleration. In this study, the Crab pulsar is observed using the $327 \mathrm{MHz}$ radio telescope at the Toyokawa Observatory of the Institute for Space-Earth Environmental Research of Nagoya University to investigate the coronal plasma density profile for radial distances between 5 and 60 solar radii at the SC24/25 minimum. We derive the dispersion measures (DMs) that represent the integration of plasma density along the line of sight (LOS) for giant radio pulses of the Crab pulsar. We find that the observed DMs increased above the interstellar background level when the LOS for the Crab pulsar approached the Sun in mid-June 2018 and 2019. This increase in DM is attributed to the effect of the coronal plasma. We determine the plasma density distribution by fitting a spherically symmetric model to the observed DM data. The flat radial slopes of the best-fit model are consistent with pulsar observations in the low-activity periods of past SCs, and they are attributed to the effect of the coronal hole over the south pole of the Sun. Our results show that the density level near the Sun is similar to those observed in the low activity periods of past SCs, implying recovery of the coronal plasma density from a significant reduction at the SC23/24 minimum.
\end{abstract}

Keywords Solar corona $\cdot$ Pulsar $\cdot$ Solar wind $\cdot$ Solar cycle

\section{Introduction}

Improved knowledge of the plasma density distribution near the Sun is crucial to gain a better understanding of the physical processes of solar wind acceleration (e.g. Muhleman and

M. Tokumaru

tokumaru@isee.nagoya-u.ac.jp

1 Institute for Space-Earth Environmental Research, Nagoya University, Nagoya, Japan

2 Present address: Sky Perfect JSAT Corporation, Tokyo, Japan

3 Present address: TOBILA Systems, Nagoya, Japan

4 Usuda Deep Space Center, Japan Aerospace Exploration Agency, Saku, Japan

5 Institute for Cosmic Ray Research, University of Tokyo, Chiba, Japan 
Anderson, 1981; Wexler et al., 2019a). To this end, in situ plasma observations in close proximity to the Sun are being conducted by the Parker Solar Probe (Fox et al., 2016) and Solar Orbiter (Müller et al., 2020); however, the radial distance range within 10 solar radii $\left(R_{S}\right)$ remains inaccessible to the direct method. The plasma density observations for this region have been conducted using remote sensing techniques, such as white-light brightness (Allen, 1947; Newkirk, 1961; Saito, Poland, and Munro, 1977; Mancuso and Garzelli, 2013), solar radio emissions (Leblanc, Dulk, and Bougeret, 1998; Mercier and Chambe, 2015), interplanetary spacecraft beacons (Stelzried et al., 1970; Tyler et al., 1977; Muhleman, Esposito, and Anderson, 1977; Edenhofer et al., 1977; Esposito, Edenhofer, and Lueneburg, 1980; Muhleman and Anderson, 1981; Bird et al., 1994; Wexler et al., 2019a,b), and pulsar dispersion measures (Counselman and Rankin, 1972, 1973; Weisberg et al., 1976; Cognard et al., 1996; Smirnova, Chashei, and Shishov, 2009; Tokumaru et al., 2020; Tiburzi et al., 2021). Remote sensing measurements of the plasma density using the white-light brightness are limited to a range within a few solar radii because the white-light diminishes rapidly with radial distance. In contrast, remote sensing techniques that use radio waves can determine the plasma density over larger distances, from a few to a few tens of solar radii, which is more important for unraveling the driving mechanisms of the solar wind. Radio-sounding measurements performed using spacecraft beacons and pulsar emissions are useful because they provide reliable estimates of integrated plasma density unambiguously. The plasma density determined using solar radio emissions depends on the assumption of a generation mechanism, which can introduce some uncertainties in the derived densities. Although spacecraft beacon measurements allow precise determination of the plasma density because of their excellent signal-to-noise ratio $(\mathrm{S} / \mathrm{N})$, the opportunities for using this approach are considerably limited due to the shortage of available interplanetary missions. In contrast, pulsars are distributed in space more abundantly than interplanetary spacecraft if a radio telescope with good sensitivity is available; therefore, the plasma density near the Sun can be investigated more frequently. Regular pulsar dispersion measurements are useful for fully clarifying the plasma density distribution near the Sun, which evolves with the solar cycle (SC). However, the integrated plasma density derived from pulsar dispersion measurements contains the contribution of the interstellar medium as well as that of the interplanetary medium, and the removal of the former is key for addressing the density distribution of the solar plasma from pulsar dispersion measurements.

In an earlier study (Tokumaru et al., 2020; hereinafter referred to as Paper I), we determined the plasma density distribution of the solar corona based on the observations of the Crab pulsar (Staelin and Reifenstein, 1968), whose line of sight (LOS) approaches the Sun as close as $5 R_{S}$ in mid-June. We derived the dispersion measure (DM), which represents the integration of the electron density along the LOS, from the Crab pulsar observations in mid-June 2018. The DM is defined as

$$
\mathrm{DM}=\int_{0}^{L} N_{e} d s,
$$

where $N_{e}, s$, and $L$ denote the electron density, distance along the LOS, and distance to the pulsar, respectively. We removed the contribution of the interstellar medium from the observed DM (the interstellar background level) to detect the enhancement in DM caused by the coronal plasma. In Paper I, the interstellar background level was determined by connecting two DMs taken 15 days before or after the closest approach to the Sun; these DMs correspond to radial distances of 52 and $56 R_{S}$, respectively. These background level observations are sufficiently far from the Sun; however, the number of observations remains 
small, and the estimation error of the background level cannot be evaluated. This affects the density profile deduced from the pulsar dispersion measurements.

This article presents a follow-up study of Paper I. We analyze Crab pulsar observations taken at the Toyokawa Observatory for the period between June 2018 and March 2020 to investigate the plasma density distribution for radial distances between 5 and $60 R_{S}$. In this study, accurate assessment of the interstellar background level is a key issue. Recently, Tiburzi et al. (2021) developed a new scheme to discriminate between the interstellar and solar contributions to the observed DMs. In this scheme, a cubic polynomial was used to model the DM variations due to the interstellar medium. The utility of this scheme was demonstrated from pulsar timing observations; nevertheless, we employed a simple method to determine the interstellar background level because its variability, revealed from our observations, was rather small during the analyzed period. We determined the background level from Crab pulsar observations over two long periods when the solar elongation exceeded $90^{\circ}$. This improves the accuracy of the background level estimation compared with that of our earlier study (Tokumaru et al., 2020). Following Paper I, we derived DMs from Crab pulsar observations for giant pulses (GPs) with an excellent S/N ratio (Heiles and Campbell, 1970; Staelin, 1970), and we fit a coronal density model to the increase in the DM observed during the closest approach to the Sun. We improved the estimation error of the best-fit model using a different threshold value for GP selection.

The remainder of this paper is organized as follows. In Section 2, we describe the Crab pulsar observations at the Toyokawa Observatory for the period between June 2018 and March 2020. In Section 3, we present the determination of the DMs for the analyzed period. In Section 4, we derive the radial variation of the increase in DM $(\Delta \mathrm{DM})$ and determine the best-fit model of the coronal plasma density from the observed $\Delta$ DMs. In Section 5, we compared the observed $\Delta \mathrm{DMs}$ with the white-light observations to examine the effect of longitude and latitude variations in the solar corona. In Sections 6 and 7, we discuss and summarize the results.

\section{Observations}

We have conducted observations of the Crab pulsar PSR B0531+21 at a frequency of $327 \mathrm{MHz}$ since September 2017 using a radio telescope called the Solar Wind Imaging Facility Telescope (SWIFT; Tokumaru et al., 2011) at the Toyokawa Observatory of the Institute for Space-Earth Environmental Research (ISEE) of Nagoya University. Detailed descriptions of the Crab pulsar observations at Toyokawa have been presented in Paper I (Tokumaru et al., 2020). The Crab pulsar observations were performed once per day for 6 or 8 minutes around the meridian transit of the source because of the performance of SWIFT. The analyzed period of this study was from June 2018 to March 2020. Although the observations for June 2018 were reported in Paper I, they were reanalyzed in this study using a different interstellar background level and a different threshold level for selecting the GPs.

Figure 1 shows the solar elongation angle of the Crab pulsar for the period between 15 May 2018 and 31 March 2020, which corresponds to MJD (modified Julian date) 58253 and 58939 , respectively. The LOS of the Crab pulsar is closest to the Sun in mid-June and farthest from it in mid-December every year. The Crab pulsar observations at Toyokawa were performed basically daily when the solar elongation angle exceeded $90^{\circ}$. These observations were used to determine the interstellar background level. Hereinafter, periods between September 2018 and March 2019 and between September 2019 and March 2020 are referred 
Figure 1 Time variation of the solar elongation angle for the Crab pulsar. Periods A, B, O1, and $\mathrm{O} 2$ are indicated by horizontal bars. In this and other figures, dates are indicated as year/month/day.

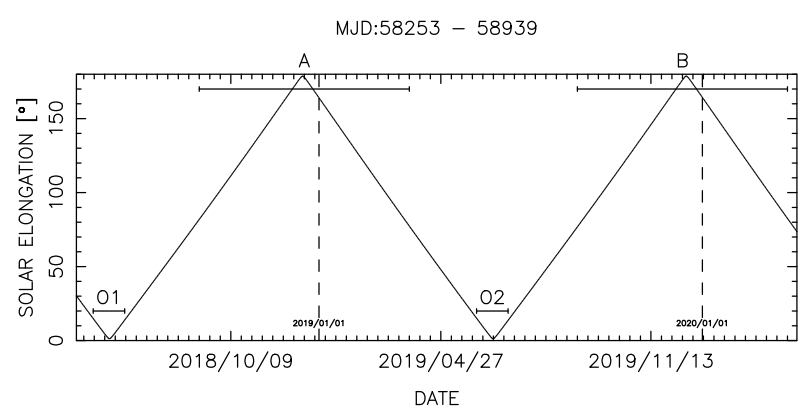

to as Periods A and B, respectively. For a solar elongation angle less than $90^{\circ}$, the observation time for the Crab pulsar was restricted considerably because interplanetary scintillation (IPS) observations of the solar wind are prioritized at Toyokawa. The Crab pulsar observations were performed daily for a 6- or 7-day period around the closest approach and for a few days before or after that period. The periods around the closest approach in 2018 and 2019 are referred to as Periods O1 and O2, respectively. During these periods, the observed DMs are expected to increase due to the effect of the coronal plasma.

\section{DM Measurements}

We first identified GPs with a $\mathrm{S} / \mathrm{N}>5$ from Crab pulsar observations at Toyokawa; then, we determined a DM for each GP with an extremely high $\mathrm{S} / \mathrm{N}$ by searching for a value that optimized the pulse height retrieved by the coherent dispersion of the received signals. In Paper I, S/N > 15 was used as a criterion to select the GPs. We calculated the mean and standard error of DMs when the number of GPs $\left(N_{G P}\right)$ obtained for a given day was greater than three and used them as the DM data in this study. This method was the same as that used in Paper I. The reliability of the DM data depends on $N_{G P}$, which significantly depends on the GP intensity, i.e. on the $\mathrm{S} / \mathrm{N}$. A higher $\mathrm{S} / \mathrm{N}$ yields a more reliable determination of DM for an individual GP; however, it results in a smaller $N_{G P}$ available in a day. Furthermore, the occurrence of GPs exhibits a significant variability on a timescale of 10 days or longer. Therefore, it is necessary to consider the threshold level of the $\mathrm{S} / \mathrm{N}$ for data selection carefully, particularly for an infrequent occurrence of GPs.

\subsection{DMs for Periods $A$ and $B$}

Figure 2 shows the number of GPs with a $\mathrm{S} / \mathrm{N}>5$ and $\mathrm{S} / \mathrm{N}>15$ identified from Crab pulsar observations at Toyokawa for Periods A and B. The occurrence of GPs in Period A exhibits large variability; $N_{G P}$ is small until January 2019 for GPs with $\mathrm{S} / \mathrm{N}>15$, whereas it increases to continuously satisfy the criterion for valid DM data in the period between 22 January and 12 March 2018 (Figure 2a). In contrast, an abundant number of GPs appears in Period B, and $N_{G P}$ for GPs with $\mathrm{S} / \mathrm{N}>15$ satisfies the criterion for valid DM data throughout that period (Figure 2b). In this study, we derived the DM data for every day between 22 January and 12 March 2018 (for Period A) and between 4 September 2019 and 21 March 2020 (for Period B).

Figure 3 shows the DM data obtained from Crab pulsar observations at Toyokawa for Period A. The number of DM data for Period A is 50, and the average value of the DMs 
(a)

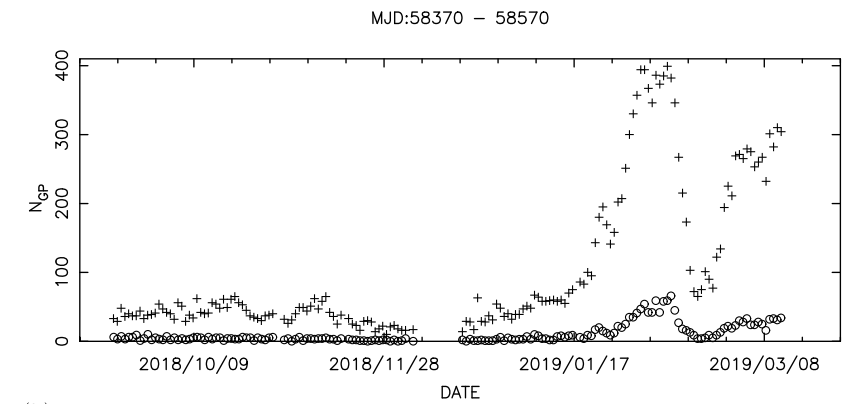

(b)

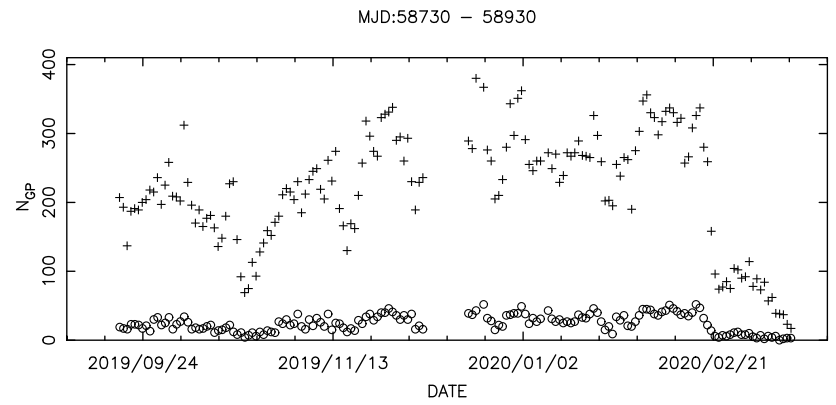

Figure 2 Time variations of $N_{G P}$ for (a) Period A and (b) Period B. Open circles and crosses correspond to GPs with $\mathrm{S} / \mathrm{N}>15$ and $\mathrm{S} / \mathrm{N}>5$, respectively.

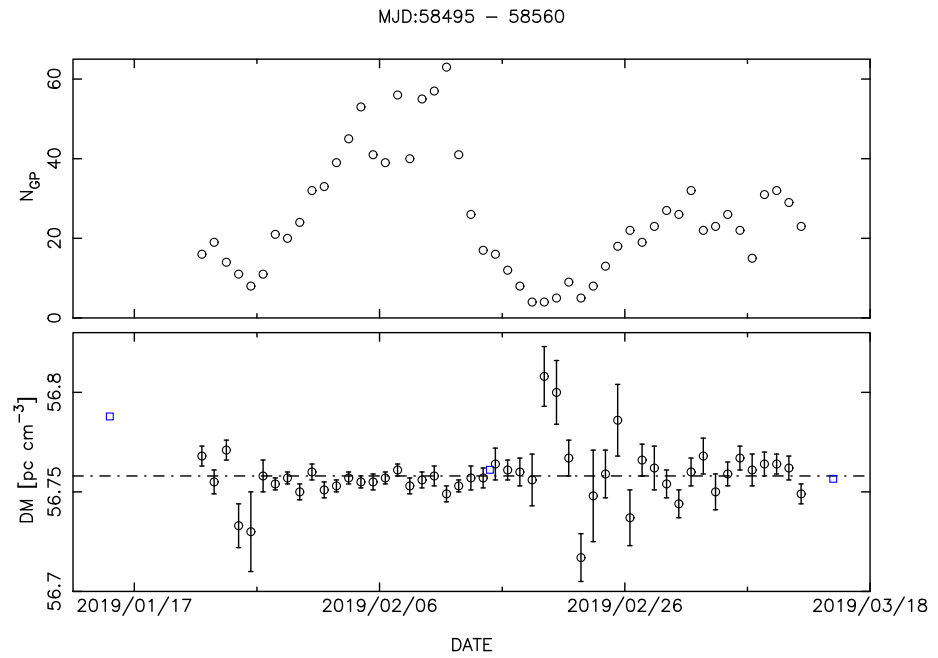

Figure 3 Time variations of (upper panel) $N_{G P}$ and (lower panel) DMs for Period A. Open circles and squares in the lower panel denote DMs at the Toyokawa and Jodrell Bank Observatories, respectively. The dash-dotted line represents the average value of the Toyokawa DM data. 
MJD:58730 - 58910

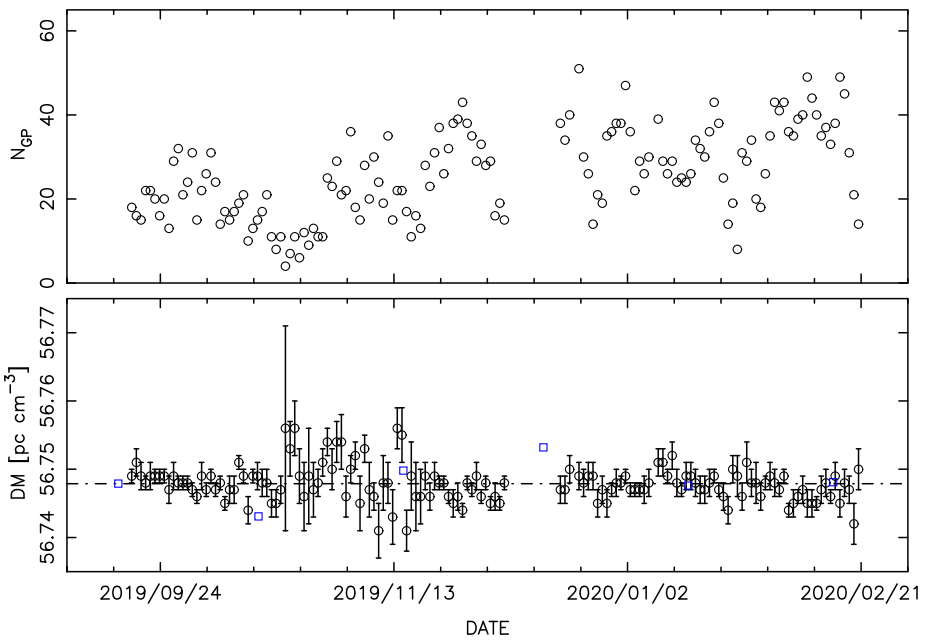

Figure 4 Time variations of (upper panel) $N_{G P}$ and (lower panel) DMs for Period B. The format is the same as that in Figure 3.

is $56.758 \pm 0.014 \mathrm{pc} \mathrm{cm}^{-3}$. The DM data do not significantly deviate from the average value; the standard errors are small except for two short periods: 25 - 26 January and 20 - 26 February 2019. Relatively large $\left(\approx \pm 0.05 \mathrm{pc} \mathrm{cm}^{-3}\right)$ excursions of the DM data and an increase in the standard errors are observed during the two periods. As $N_{G P}$ shows minima for the two periods, the large excursions and increased standard errors are ascribed to the effect of the reduced number of data. However, the excursions appear to occur randomly around the average value, and no systematic tendency is observed even for the two periods. The DM data obtained at the Jodrell Bank Observatory (Lyne, Pritchard, and Graham Smith, 1993) in Period A are indicated in Figure 3. The Jodrell Bank data taken on 15 February and 15 March 2019 show good agreement with the average value of the Toyokawa data. Although the Jodrell Bank DM value on 15 January 2019 is higher than the average value of the Toyokawa data, it is not very different. Therefore, we consider the average value of $56.758 \pm 0.014 \mathrm{pc} \mathrm{cm}^{-3}$ as a reliable estimate of the DM in Period A. This value is almost the same as the average value of the DM data taken between 19 September 2017 and 15 March 2018 (hereinafter, Period C): $56.759 \pm 0.003 \mathrm{pc} \mathrm{cm}^{-3}$ (Tokumaru et al., 2020).

Figure 4 shows the DM data obtained for Period B. A total of $114 \mathrm{DM}$ data are available for this period; the average value of DMs is $56.748 \pm 0.003 \mathrm{pc} \mathrm{cm}^{-3}$, which is slightly smaller than that for Period A. The standard errors of the DM data for Period B are also smaller than those for Period A owing to the larger number of data. The DM data for Period B are almost constant, similarly to Period A, whereas the fluctuations of the DM data are enhanced for the period 19 October-13 November 2019. The enhanced fluctuations are attributed to a decrease in $N_{G P}$. Furthermore, the Toyokawa DM data are in good agreement with those of Jodrell Bank, and therefore the average value of $56.748 \pm 0.003 \mathrm{pc} \mathrm{cm}^{-3}$ is considered as a reliable estimate of the DM for Period B. The difference in the average value between Periods A and B may be ascribed to the long-term variation of the interstellar medium. 


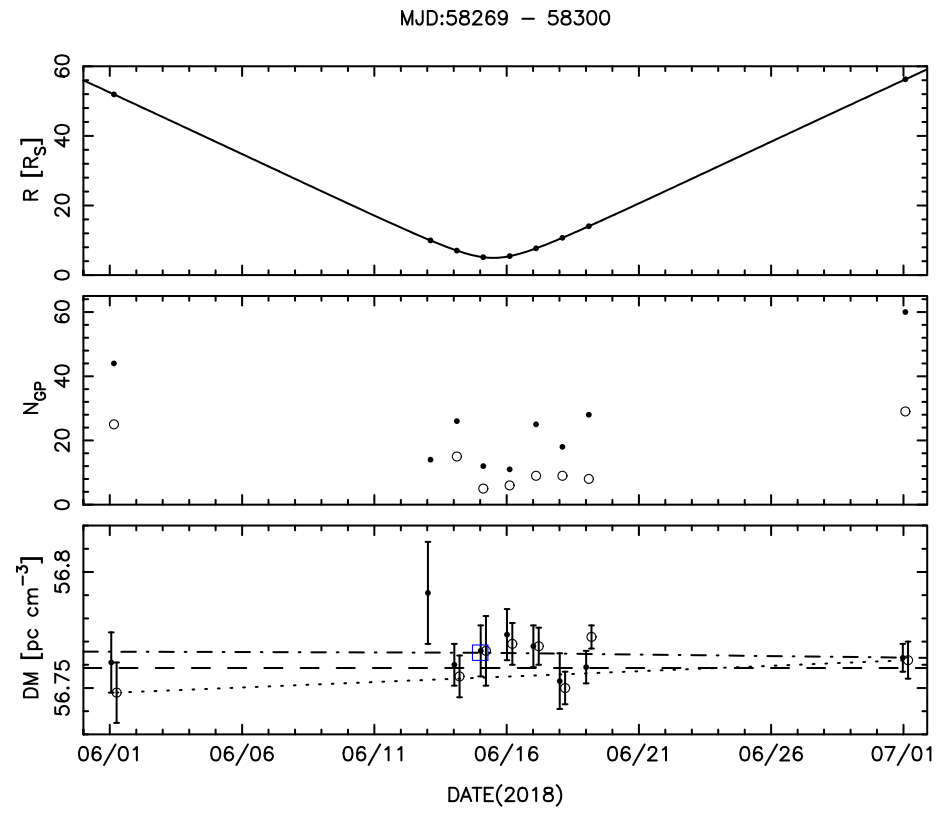

Figure 5 Time variations of (top panel) the solar offset distance of the LOS $(R)$, (middle panel) $N_{G P}$, and (bottom panel) DM for Period O1. Open and solid circles correspond to the data for GPs with S/N $>15$ and $\mathrm{S} / \mathrm{N}>10$, respectively. The open square indicates DM data at the Jodrell Bank Observatory, and the dashdotted line connects adjacent Jodrell Bank data. The background level derived from the DM data for Period $\mathrm{A}$ is indicated by the dashed line. The dotted line shows the background level used in Paper I.

Table 1 DM data for Period O1.

\begin{tabular}{|c|c|c|c|c|c|c|}
\hline \multirow[t]{2}{*}{ Date } & \multirow[t]{2}{*}{ MJD } & \multirow[t]{2}{*}{$R\left[R_{S}\right]$} & \multicolumn{2}{|c|}{$\mathrm{S} / \mathrm{N}>15$} & \multicolumn{2}{|c|}{$\mathrm{S} / \mathrm{N}>10$} \\
\hline & & & $N_{G P}$ & $\mathrm{DM}\left[\mathrm{pc} \mathrm{cm}{ }^{-3}\right]$ & $N_{G P}$ & $\mathrm{DM}\left[\mathrm{pc} \mathrm{cm}{ }^{-3}\right]$ \\
\hline 20180601 & 58270 & 51.9 & 25 & $56.748 \pm 0.013$ & 44 & $56.761 \pm 0.013$ \\
\hline 20180613 & 58282 & 10.0 & 3 & $56.818 \pm 0.051$ & 14 & $56.791 \pm 0.022$ \\
\hline 20180614 & 58283 & 7.0 & 15 & $56.755 \pm 0.009$ & 26 & $56.760 \pm 0.009$ \\
\hline 20180615 & 58284 & 5.1 & 5 & $56.766 \pm 0.015$ & 12 & $56.766 \pm 0.011$ \\
\hline 20180616 & 58285 & 5.4 & 6 & $56.769 \pm 0.009$ & 11 & $56.773 \pm 0.011$ \\
\hline 20180617 & 58286 & 7.7 & 9 & $56.768 \pm 0.008$ & 25 & $56.768 \pm 0.009$ \\
\hline 20180618 & 58287 & 10.7 & 9 & $56.750 \pm 0.007$ & 18 & $56.753 \pm 0.012$ \\
\hline 20180619 & 58288 & 14.0 & 8 & $56.772 \pm 0.005$ & 28 & $56.759 \pm 0.007$ \\
\hline 20180701 & 58300 & 56.3 & 29 & $56.762 \pm 0.008$ & 60 & $56.763 \pm 0.006$ \\
\hline
\end{tabular}

\subsection{DMs for Periods 01 and 02}

Figure 5 and Table 1 show the DM data derived for Period O1 when the LOS of the Crab pulsar approached the Sun in 2018. Even though the DM data for Period O1 were presented in Paper I, the data of this study include values determined for GPs with $\mathrm{S} / \mathrm{N}>10$ as well as those with $\mathrm{S} / \mathrm{N}>15$. Data of the solar elongation distance of the $\operatorname{LOS}(R)$ and $N_{G P}$ are also indicated in the figure and the table. As reported in Paper I, the occurrence of GPs 
significantly diminished in Period O1, which degraded the accuracy of the DM data. In this study, we improved the accuracy of the DM data for Period O1 by increasing the number of data. As shown in Figure 5, the number of GPs for $\mathrm{S} / \mathrm{N}>10$ is approximately twice greater than that for $S / N>15$. This results in a larger number of $D M$ data for $S / N>10$ than for $\mathrm{S} / \mathrm{N}>15$ : 9 and 8, respectively. However, it is not obvious that the DM data for $\mathrm{S} / \mathrm{N}>10$ have better accuracy than those for $\mathrm{S} / \mathrm{N}>15$ because the accuracy of an individual $\mathrm{DM}$ estimate can be worse due to the relative increase in noise. The daily means and standard errors of Period O1 DM data for $\mathrm{S} / \mathrm{N}>10$ are almost the same as those for $\mathrm{S} / \mathrm{N}>15$, except for those taken on 1 and 19 June 2018. The average values of the standard errors for $\mathrm{S} / \mathrm{N}>10$ and $\mathrm{S} / \mathrm{N}>15$ are $0.011 \mathrm{pc} \mathrm{cm}^{-3}$ and $0.009 \mathrm{pc} \mathrm{cm}^{-3}$, respectively. The slightly larger average value of the standard error for $\mathrm{S} / \mathrm{N}>10$ is ascribed to the contribution of the DM data on 13 June 2018. A marked enhancement in the DM is observed on this day for the $S / N>10$ data. The DM data for $\mathrm{S} / \mathrm{N}>15$ show a similar enhancement, although the number of GPs $\left(N_{G P}=3\right)$ is considered insufficient for this to be a valid data point. If the value of the DM data for $\mathrm{S} / \mathrm{N}>10$ on 13 June 2018 is ignored, the average value of the standard error for $\mathrm{S} / \mathrm{N}>10$ is $0.0097 \mathrm{pc} \mathrm{cm}^{-3}$ and results in slightly smaller estimation errors of the model fit in percentage, while the errors are still large. Although the error bar of this data point is relatively large, there is no sound reason to exclude it because the number of GPs for $\mathrm{S} / \mathrm{N}>10$ on this day is abundant enough to provide a reliable value of the DM; therefore, we included this in further analysis.

We assume that the interstellar background level during Period $\mathrm{O} 1$ is given by the average value of DM estimates for the Periods $\mathrm{A}$ and $\mathrm{C}$, as Period $\mathrm{O} 1$ corresponds to the midpoint between Periods A and C. Such a linear interpolation of the DM data is considered appropriate as a first-order approximation for estimating the interstellar background level because the difference in the DM estimates between Period A and C is insignificant. In Paper I, the interstellar background level was determined by connecting two values of the DM data taken on 1 June and 1 July 2018. The background level of Paper I is similar but slightly lower than that in this study (Figure 5). The DM data collected on days around the closest approach to the Sun are higher or nearly equal to the interstellar background level except for those obtained on 18 June 2018. This enhancement in the DM is ascribed to the effect of the solar corona. The DM data obtained at Jodrell Bank are quite consistent with our DM data; they are slightly above the interstellar background level. This fact supports the reliability of our DM data.

The DM data for Period O2 are shown in Figure 6 and Table 2, their formats are the same as the ones of Figure 5 and Table 1, respectively. The average value of DM estimates for Periods A and B is used as the interstellar background level of Period O2. While the difference in the DM estimates between Periods A and B is larger than that between Periods $\mathrm{A}$ and $\mathrm{C}$, it is still small; therefore, the linear interpolation method is considered applicable for the determination of the interstellar background level in Period O2. As is the case with Period O1, $N_{G P}$ for Period O2 shows a significant decrease as the LOS of the Crab pulsar approaches the Sun. The cause for this decrease is not yet fully understood; however, we consider that it is unlikely to be due to the intrinsic variation of the GP occurrence and more likely to be due to an instrumental effect. The GPs with $\mathrm{S} / \mathrm{N}>10$ yield more DM data than those with $\mathrm{S} / \mathrm{N}>15$ : 9 and 8, respectively, because of their abundant occurrence. The $\mathrm{DM}$ data for $\mathrm{S} / \mathrm{N}>10$ appear to be quite similar to those for $\mathrm{S} / \mathrm{N}>15$, except for that of 18 June 2019 . The standard errors for $S / N>10$ are slightly smaller than those for $S / N>15$ : the average values of the standard errors for $\mathrm{S} / \mathrm{N}>10$ and $\mathrm{S} / \mathrm{N}>15$ are $0.006 \mathrm{pc} \mathrm{cm}^{-3}$ and $0.011 \mathrm{pc} \mathrm{cm}^{-3}$, respectively. This difference is mainly caused by the DM data on 18 June 2019. When this data point is ignored, the average value of the standard errors for $\mathrm{S} / \mathrm{N}>15$ 


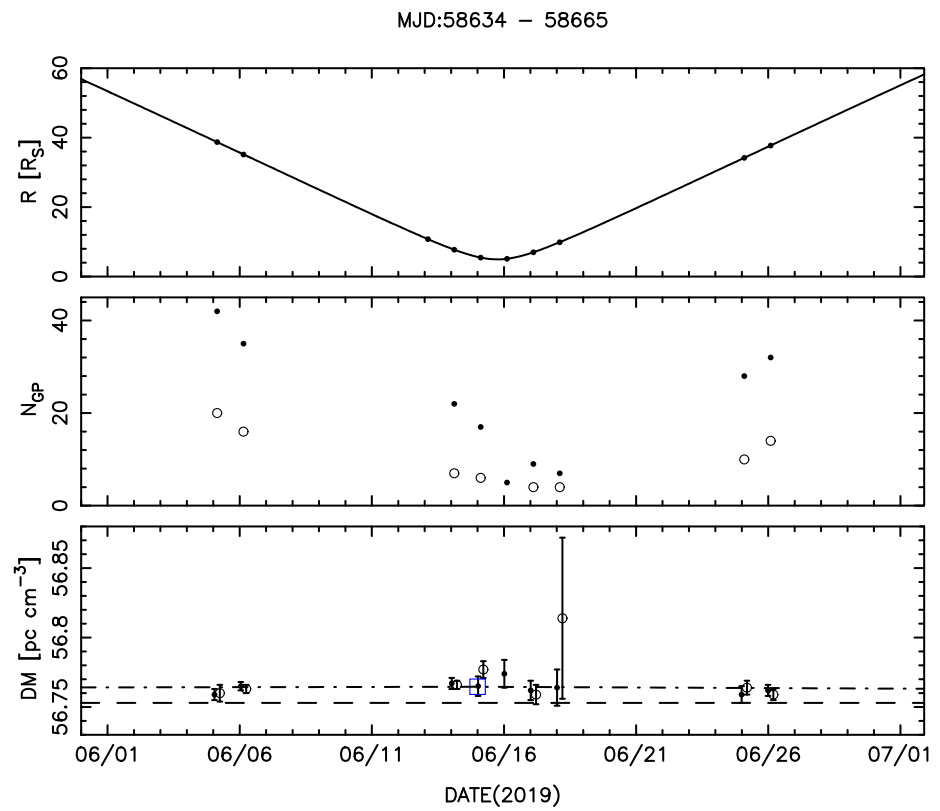

Figure 6 Time variations of (top panel) the solar offset distance of the LOS $(R)$, (middle panel) $N_{G P}$, and (bottom panel) DM for Period O2. Open and solid circles correspond to the data for GPs with $\mathrm{S} / \mathrm{N}>15$ and $\mathrm{S} / \mathrm{N}>10$, respectively. The open square indicates DM data at the Jodrell Bank Observatory, and the dashdotted line connects adjacent Jodrell Bank data. The background level given by the average value of the DM data between Periods A and B is indicated by the dashed line.

Table 2 DM data for Period O2.

\begin{tabular}{|c|c|c|c|c|c|c|}
\hline \multirow[t]{2}{*}{ Date } & \multirow[t]{2}{*}{ MJD } & \multirow[t]{2}{*}{$R\left[R_{S}\right]$} & \multicolumn{2}{|c|}{$\mathrm{S} / \mathrm{N}>15$} & \multicolumn{2}{|c|}{$\mathrm{S} / \mathrm{N}>10$} \\
\hline & & & $N_{G P}$ & $\mathrm{DM}\left[\mathrm{pc} \mathrm{cm}{ }^{-3}\right]$ & $N_{G P}$ & $\mathrm{DM}\left[\mathrm{pc} \mathrm{cm}{ }^{-3}\right]$ \\
\hline 20190605 & 58639 & 38.7 & 20 & $56.760 \pm 0.006$ & 42 & $56.759 \pm 0.004$ \\
\hline 20190606 & 58640 & 35.2 & 16 & $56.763 \pm 0.003$ & 35 & $56.765 \pm 0.003$ \\
\hline 20190614 & 58648 & 7.7 & 7 & $56.766 \pm 0.003$ & 22 & $56.767 \pm 0.004$ \\
\hline 20190615 & 58649 & 5.5 & 6 & $56.777 \pm 0.006$ & 17 & $56.765 \pm 0.007$ \\
\hline 20190616 & 58650 & 5.1 & 1 & 56.804 & 5 & $56.774 \pm 0.010$ \\
\hline 20190617 & 58651 & 7.0 & 4 & $56.759 \pm 0.007$ & 9 & $56.762 \pm 0.007$ \\
\hline 20190618 & 58652 & 9.9 & 4 & $56.814 \pm 0.058$ & 7 & $56.764 \pm 0.013$ \\
\hline 20190625 & 58659 & 34.2 & 10 & $56.764 \pm 0.005$ & 28 & $56.759 \pm 0.006$ \\
\hline 20190626 & 58660 & 37.7 & 14 & $56.759 \pm 0.004$ & 32 & $56.762 \pm 0.004$ \\
\hline
\end{tabular}

is $0.005 \mathrm{pc} \mathrm{cm}^{-3}$, which is nearly the same as that for $\mathrm{S} / \mathrm{N}>10$. This data point shows an abrupt increase in DM, similar to the DM data on 13 June 2018 for $\mathrm{S} / \mathrm{N}>10$. However, this is inconsistent with the DM data for $\mathrm{S} / \mathrm{N}>10$ on the same day. The discrepancy may be due to poor statistics for $\mathrm{S} / \mathrm{N}>15 \mathrm{DM}$ data. Nevertheless, $N_{G P}$ satisfies the criterion for valid data in this study; therefore, this DM data point was used in further analysis. We note that removal of this data point results in a model fit that is more consistent with that for $\mathrm{S} / \mathrm{N}>10$. 
Table 3 Parameters of the best-fit models.

\begin{tabular}{|c|c|c|c|c|c|c|c|}
\hline \multirow{2}{*}{$\begin{array}{l}\text { Year } \\
2018\end{array}$} & \multirow{2}{*}{$\begin{array}{l}\mathrm{S} / \mathrm{N} \\
15\end{array}$} & \multicolumn{2}{|c|}{$\alpha$ (Fractional error) } & \multicolumn{2}{|c|}{$\begin{array}{l}N_{0}\left[10^{3} \mathrm{~cm}^{-3}\right] \\
\text { (Fractional error) }\end{array}$} & \multirow{2}{*}{$\begin{array}{l}\begin{array}{l}\text { Degrees } \\
\text { of } \\
\text { freedom }\end{array} \\
6\end{array}$} & \multirow{2}{*}{$\begin{array}{l}\begin{array}{l}\text { RMS } \\
\text { residuals }\end{array} \\
0.0090\end{array}$} \\
\hline & & $4.42 \pm 6.72$ & $(152 \%)$ & $2.78 \pm 8.54$ & $(308 \%)$ & & \\
\hline 2018 & 10 & $1.50 \pm 0.98$ & $(65 \%)$ & $6.37 \pm 8.82$ & $(139 \%)$ & 7 & 0.0114 \\
\hline 2019 & 15 & $1.40 \pm 0.52$ & $(37 \%)$ & $14.4 \pm 15.9$ & $(111 \%)$ & 6 & 0.0188 \\
\hline 2019 & 10 & $1.33 \pm 0.15$ & $(11 \%)$ & $7.30 \pm 2.69$ & $(37 \%)$ & 7 & 0.0036 \\
\hline 2018-2019 & 15 & $1.39 \pm 0.59$ & $(42 \%)$ & $7.67 \pm 9.31$ & $(121 \%)$ & 14 & 0.0162 \\
\hline 2018-2019 & 10 & $1.37 \pm 0.32$ & $(23 \%)$ & $6.49 \pm 4.46$ & $(69 \%)$ & 16 & 0.0082 \\
\hline
\end{tabular}

The systematic excess in the DM relative to the background level is revealed in Figure 6; it is ascribed to the effect of the solar corona. The DM data at the Jodrell Bank Observatory are consistent with our DM data. As in the case of Period O1, the Jodrell Bank DM data for Period $\mathrm{O} 2$ are close but slightly higher than the background level, which suggests an enhancement in the DM associated with the closest approach to the Sun.

\section{Radial Variation of $\Delta \mathrm{DM}$}

We determine the difference in DM $(\triangle \mathrm{DM})$ by subtracting the background level from the $\mathrm{DM}$ data. The $\Delta \mathrm{DMs}$ from the $\mathrm{DM}$ data with $\mathrm{S} / \mathrm{N}>10$ for Periods $\mathrm{O} 1$ and $\mathrm{O} 2$ are plotted as a function of the solar elongation distance $R$ of the LOS in Figures 7a and b, respectively. We fit the spherically symmetric (electron) density model $N_{e}(r)$ given by Equation 2 to $\Delta$ DMs:

$$
N_{e}(r)=N_{0}\left(\frac{r}{r_{0}}\right)^{-\alpha},
$$

where $\alpha, r$, and $N_{0}$ denote the power-law index, radial distance, and electron density at $r_{0}$, respectively, and we assume $r_{0}=10 R_{S}$. The best-fit model for DM data with $\mathrm{S} / \mathrm{N}>10$ is indicated in each panel. The $\triangle \mathrm{DMs}$ and the density model fitted to the combined data set of Periods $\mathrm{O} 1$ and $\mathrm{O} 2$ are also shown in Figure 7c.

The parameters of the best-fit model are listed in Table 3. The best-fit parameters of the Period O1/O2/O1+O2 models are consistent with each other except for the case of $\mathrm{S} / \mathrm{N}>15$ in Period O1. The similarity of the best-fit models for 2018 and 2019 suggests that the plasma density distribution near the Sun is stable during 2018-2019, and this is consistent with the condition of the solar corona at a minimum. The model fit for $\mathrm{S} / \mathrm{N}>15$ of Period $\mathrm{O} 1$ is different from the others, although the reason for this is not fully understood. No obvious outlier is included in the DM data with $\mathrm{S} / \mathrm{N}>15$ for Period $\mathrm{O} 1$, unlike those with $\mathrm{S} / \mathrm{N}>10$. We found from simultaneous white-light observations that none of the DM data for Periods $\mathrm{O} 1$ and $\mathrm{O} 2$ are affected by coronal mass ejections (CMEs; see Section 5). Furthermore, the model fit for $\mathrm{S} / \mathrm{N}>15$ in Period $\mathrm{O} 1$ is inconsistent with the results of Paper I. The discrepancy between Paper I and this study may be due to the difference in the interstellar background level used to derive $\triangle \mathrm{DM}$; however, we cannot safely rule out the possibility of a measurement error because only two data points were used to derive the interstellar background level in Paper I. The estimation errors of the best-fit models for $\mathrm{S} / \mathrm{N}>10$ are smaller than those for $\mathrm{S} / \mathrm{N}>15$ indicating that the $\mathrm{DM}$ data with $\mathrm{S} / \mathrm{N}>10$ yield a better fit 


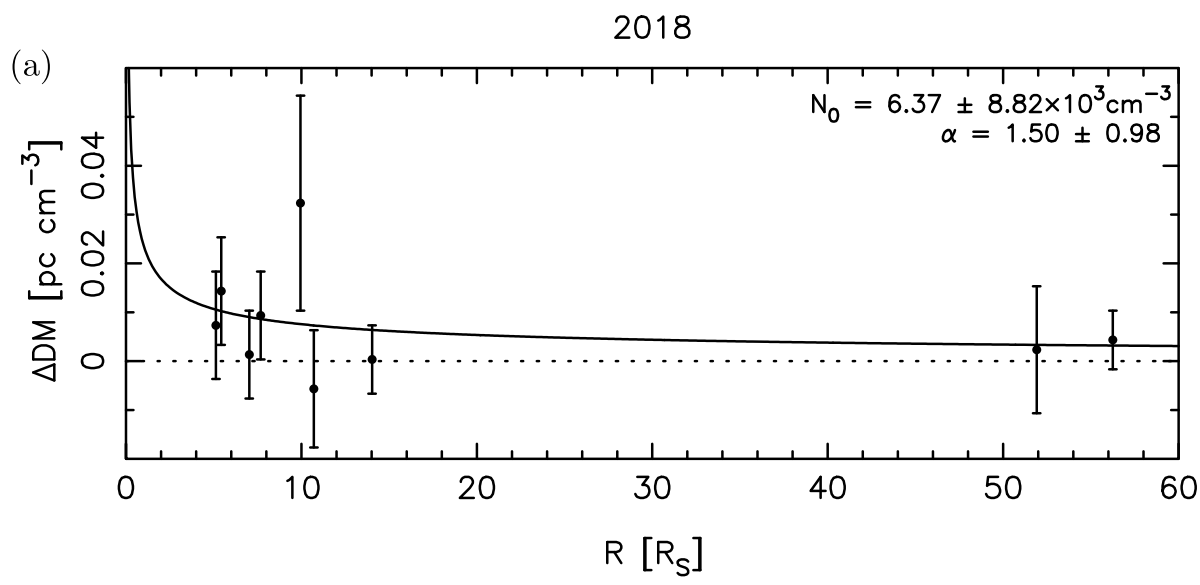

2019

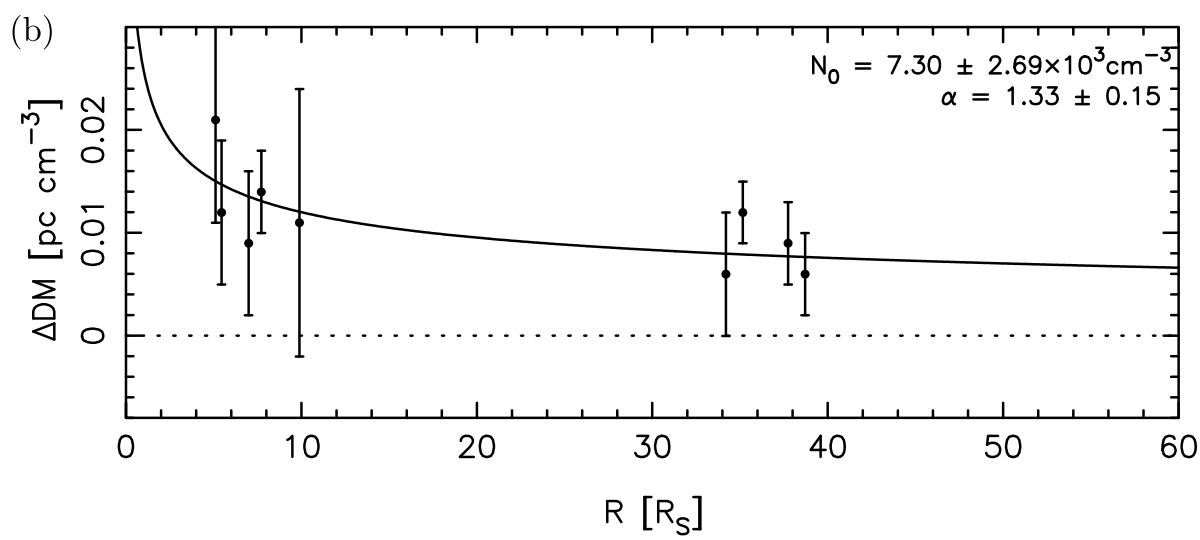

$2018-2019$

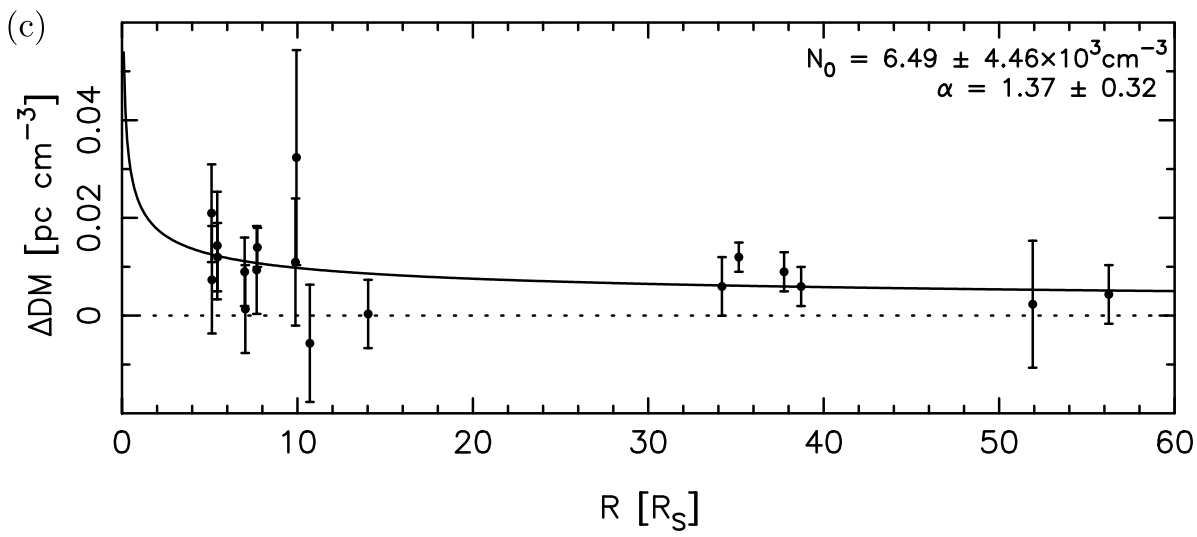

Figure $7 \Delta$ DM derived from GPs with $\mathrm{S} / \mathrm{N}>10$ plotted as a function of $R$ for (a) Period O1, (b) Period $\mathrm{O} 2$, and (c) combined Periods O1 and O2. The solid curve in each panel indicates the best-fit model. The parameters of the best-fit model are indicated at the top right of each panel. 
Figure 8 Coronal density models derived from pulsar DM observations. The solid lines correspond to models obtained at solar minima or declining phases (models a-d). The dashed lines correspond to those obtained at solar maxima (models $\mathrm{e}-\mathrm{i}$ ). The blue lines indicate those obtained in this study (models A-C). Information on models $\mathrm{a}-\mathrm{i}$ and $\mathrm{A}-\mathrm{C}$ is summarized in Table 4 .

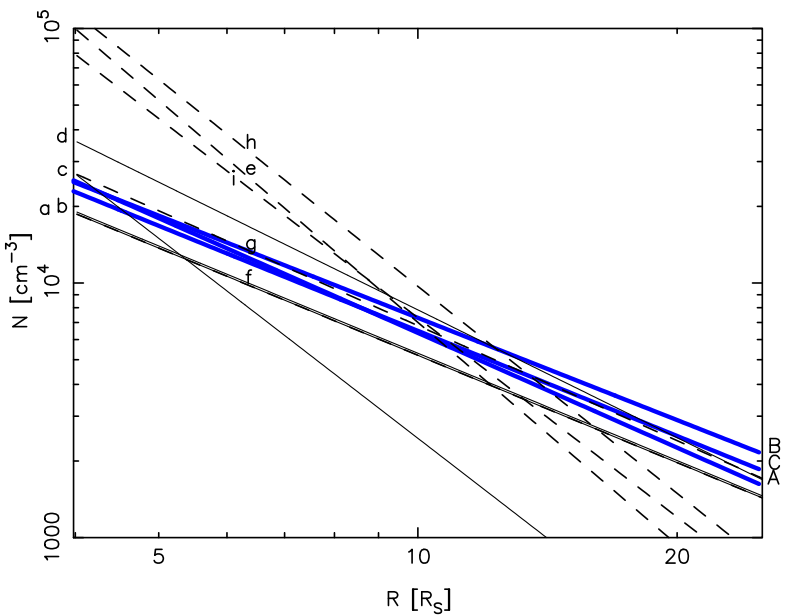

Table 4 Coronal density models derived from pulsar DM observations.

\begin{tabular}{llllll}
\hline Model & Year & Solar activity & $\begin{array}{l}N_{0} \\
{\left[10^{3} \mathrm{~cm}^{-3}\right]}\end{array}$ & $\alpha$ & References \\
\hline a & $1992-1993$ & SC22 declining phase & 5.2 & 1.4 & Cognard et al. (1996) \\
b & 1973 & SC20 declining phase & 5.3 & 1.4 & Weisberg et al. (1976) \\
c & 2005,2007 & SC23/24 minimum & 2.5 & 2.6 & Smirnova, Chashei, and Shishov \\
& & & & & $(2009)$ \\
d & 2018 & SC24/25 minimum & 7.8 & 1.7 & Paper I \\
e & 1969,1970 & SC20 maximum & 7.0 & 2.9 & Counselman and Rankin (1972) \\
f & 1971 & SC20 maximum & 5.2 & 1.4 & Counselman and Rankin (1973) \\
g & $1989-1990$ & SC22 maximum & 6.8 & 1.5 & Cognard et al. (1996) \\
h & $1990-1991$ & SC22 maximum & 9.7 & 2.7 & Cognard et al. (1996) \\
i & $1991-1992$ & SC22 maximum & 7.3 & 2.6 & Cognard et al. (1996) \\
A & 2018 & SC24/25 minimum & 6.4 & 1.5 & This article \\
B & 2019 & SC24/25 minimum & 7.3 & 1.3 & This article \\
C & 2018,2019 & SC24/25 minimum & 6.5 & 1.4 & This article \\
\hline
\end{tabular}

to the model. Therefore, we adopt the model to fit $\mathrm{S} / \mathrm{N}>10$ as the most reliable one in this study.

Figure 8 illustrates the coronal density models obtained from pulsar dispersion measurements, including those in this study. The observation years and solar activity phases corresponding to the models are indicated in Table 4. Figure 8 shows that the radial slopes of the coronal density models obtained at solar minima or declining phases of the SC (solid lines; models $\mathrm{a}-\mathrm{d}$ ), except for model $\mathrm{c}$ are flatter than those obtained at the solar maxima (dashed lines; models $\mathrm{e}-\mathrm{i}$ ). Furthermore, the plasma density at $5 R_{S}$ in the low solar activity periods is two or three times lower than that in the solar maximum periods. These differences are ascribed to the effect of the low-density region associated with the coronal hole at high latitudes. It is known that coronal holes develop over the poles in the low solar activity period. The radial slope of model c, which corresponds to the SC23/24 minimum, is as steep as that at the solar maxima; however, the plasma density at $5 R_{S}$ is as low as 
those in the low-activity periods. Thus, the plasma density of model c drops significantly at larger distances. This is consistent with the marked decline in the solar wind density reported for the SC23/24 minimum (McComas et al., 2008). The models obtained from this study (denoted as A, B, and C in the figure) are consistent with those from earlier studies for the low-activity periods of past cycles (models a and $b$ ). This suggests that the coronal density distribution at SC24/25 minimum remains unchanged from that in the low-activity periods despite a marked decline in the solar activity in SC24.

\section{Comparison with LASCO Observations}

The plasma distribution in the corona deviates from spherical symmetry in the low solar activity period like in the analyzed period of this study (SC24/25 minimum); this is due to the development of rarefied regions associated with polar coronal holes. The latitude structure of the solar corona significantly affects the density models derived in this study because the heliographic latitude of the LOS for the Crab pulsar varies with the solar elongation distance. This is a common issue for radio-sounding observations using a single LOS. The projected location of any radio source moves from low to high latitudes as it approaches the Sun, which means low-latitude data points are biased to larger distances, and high-latitude data points are available only in the region close to the Sun. This makes establishing of the coronal or solar wind model for each regime rather challenging. A density model with latitude dependence is useful for analyzing Crab pulsar observations at SC24/25 minimum. However, the DM data obtained from this study are too few to address the latitude and radial dependence of the plasma density by fitting the model. Instead, we examine the effect of the latitude structure of the plasma density on Crab pulsar observations by comparing the DM data with the Large Angle and Spectrometric Coronagraph (LASCO) C3 coronagraph observations (Brueckner et al., 1995) on board the Solar and Heliospheric Observatory (SOHO).

Figure 9 shows the synoptic maps of the white-light corona produced from LASCO C3 observations at $5 R_{S}$ for (a) CR 2205 and (b) 2218, corresponding to Periods O1 and O2, respectively. The LASCO C3 synoptic maps are available at different heights up to $25 R_{S}$. The coronal features revealed in those maps at different heights do not show significant radial evolution, while they become faint and noisy for $>15 R_{S}$. In this study, the synoptic map at $5 R_{S}$ with a high-contrast image was employed as representative one to investigate the effect of the latitude/longitude structure of the corona. It should be noted that LASCO C3 observations contain a projection effect; therefore, the appearance of some coronal structures is distorted in the maps. The projected locations of the point closest to the Sun (called "P-point") for the LOS of Crab pulsar observations are indicated in the maps. The density variation along the LOS sharply peaks at the P-point because of the radial dependence (Figure 9 of Paper I); therefore, the contribution from the P-point dominates the DM data. This effect becomes remarkable when the P-point is located in the immediate proximity to the Sun, as is the case of Crab pulsar observations in mid-June. While an analysis using the P-point is useful to gain a quick insight into the density profile, it should be noted that contributions can be expected from other portions of the LOS, and they may become noticeable, for instance, when the other portions of the LOS lie above a coronal streamer.

In this study, we employed the P-point as a reference point for our DM data. As shown in Figure 9, the P-point moves from the Equator to the South Pole as the LOS approaches the Sun. Despite such a large movement in latitude, the P-point is located in the low-brightness region for most of the period. This suggests that the spherically symmetric model used in this study is sufficient as a first-order approximation. Nevertheless, the best-fit models are 
(a)

LASCO(C3/W/O5 R $\left.\mathrm{R}_{\mathrm{S}}\right)$

CARRINGTON ROTATION: 2205

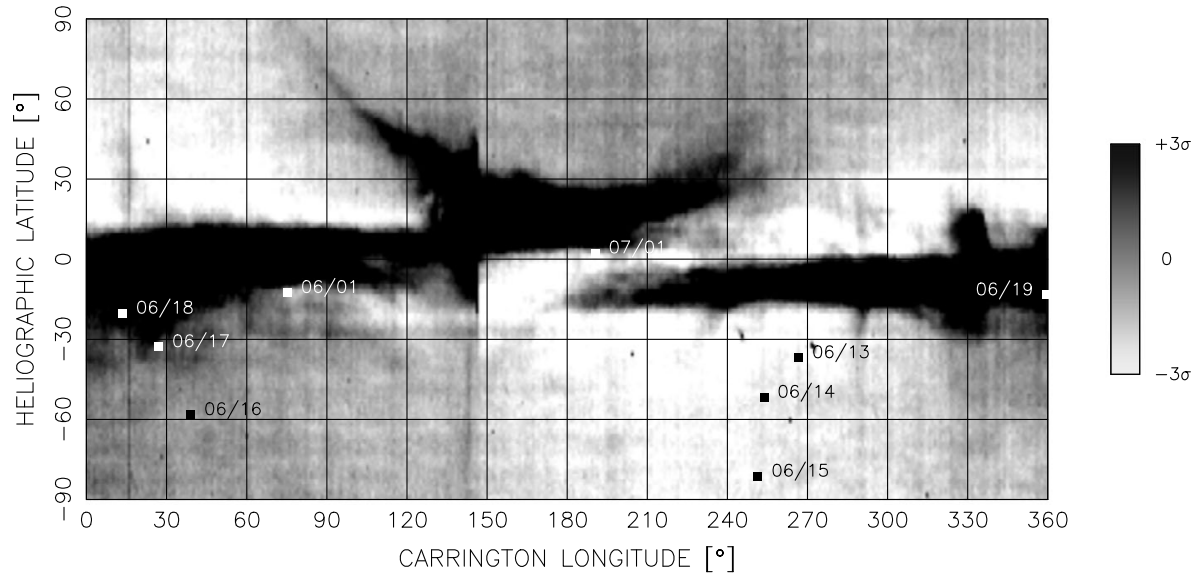

(b) $\quad \mathrm{LASCO}\left(\mathrm{C} 3 / \mathrm{W} / 05 \mathrm{R}_{\mathrm{S}}\right)$

CARRINGTON ROTATION: 2218

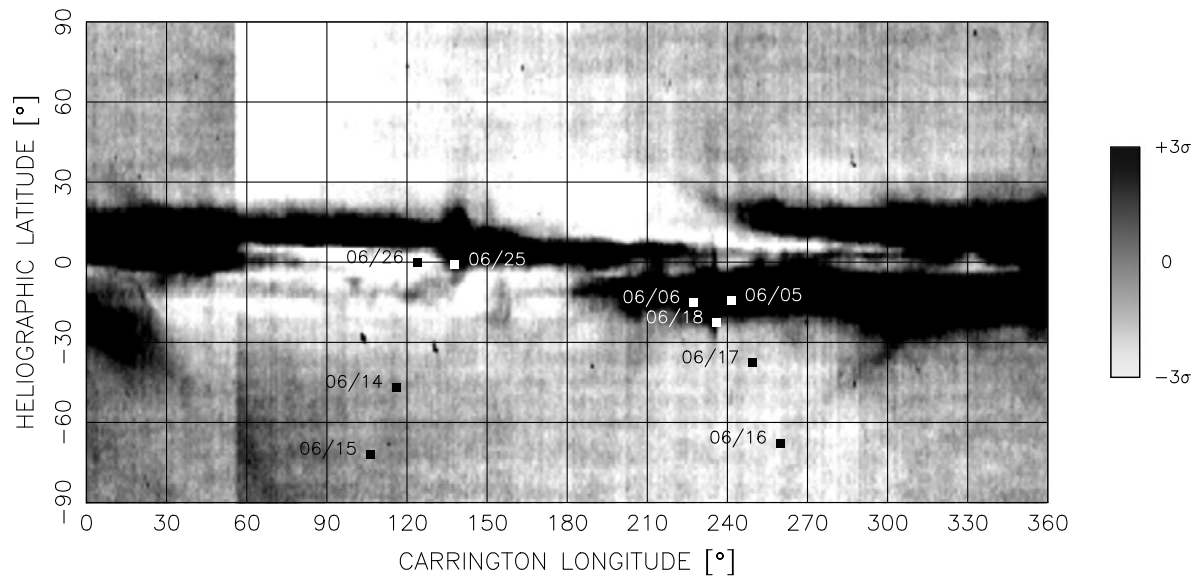

Figure 9 Synoptic Carrington maps of the white-light corona at $5 R_{S}$ derived from LASCO C3 observations on the west limb for (a) CR 2205 and (b) CR 2218. The solid blue squares in each map indicate the projected locations of the P-point on the LOS of the Crab pulsar for Periods O1 or O2.

likely to be significantly affected by the effect of the latitude structure of the solar corona because the P-point is located at the equatorial bright region when the LOS is far from the Sun. Figure 10 shows the $\triangle \mathrm{DM}$ and the white-light brightness at the P-point for CR 2205 and CR 2218. The DM data include the radial variation as well as longitude and latitude variations. The white-light brightness at the P-point is low during the closest approach, and it is enhanced as the LOS moves away from the Sun; this corresponds to the movement of the P-point from the rarefied region associated with the polar coronal hole to the dense region associated with the equatorial streamer and vice versa. This change greatly suppresses the increase in DM at the closest approach, and it results in a flatter slope of the best-fit model 
Figure 10 Time variations of (square) white-light brightness and (circle) $\triangle \mathrm{DM}$ for (a) $\mathrm{CR}$ 2205 and (b) CR 2218. (a) CR2205

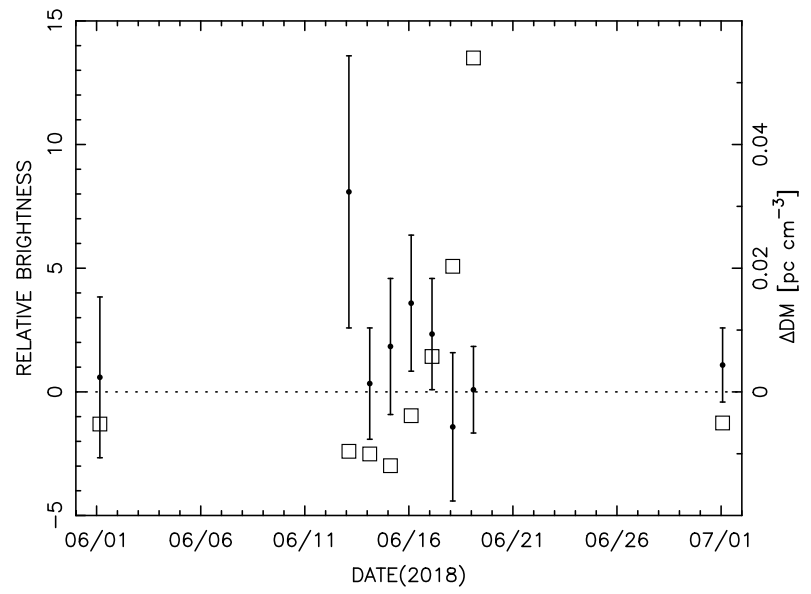

(b)

CR2218

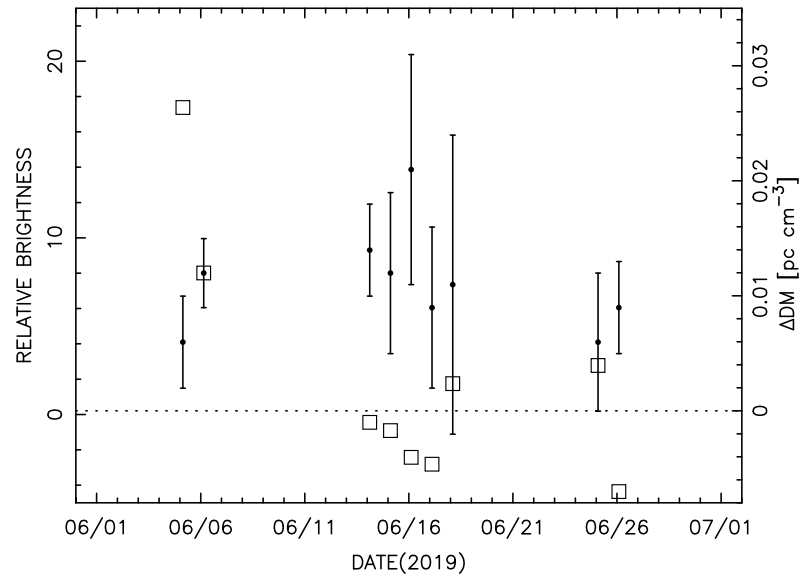

of the coronal density. Although the P-points for 1 June and 1 July 2018 (Period O1) and 26 June 2019 (Period O2) are located at the Equator, their white-light brightness levels are as low as those for the period around the closest approach. This is ascribed to the longitudinal structure of the equatorial streamer.

Figures 11 and 12 show LASCO C3 images obtained daily during Periods O1 and O2. The LASCO C3 images collected near the observation time of Crab pulsar data are selected here. The LOSs for the Crab pulsar at the beginning and the end of the observation periods are located beyond the field of view of LASCO C3 $\left(30 R_{S}\right)$; therefore, the images for those days are not shown in the figures. The movement of the LOS from the dark region over the South Pole to the bright region at the Equator is revealed in the figures; the LOSs on 18 and 19 June 2018 and 18 June 2019 intersected equatorial streamers associated with dense plasmas. The figures also display that none of the LOSs intersected transient features are associated with CMEs. Although a CME was found on 15 June 2018 over the west limb near the Equator, the LOS for the Crab pulsar was located at the South Pole, sufficiently 
(a)

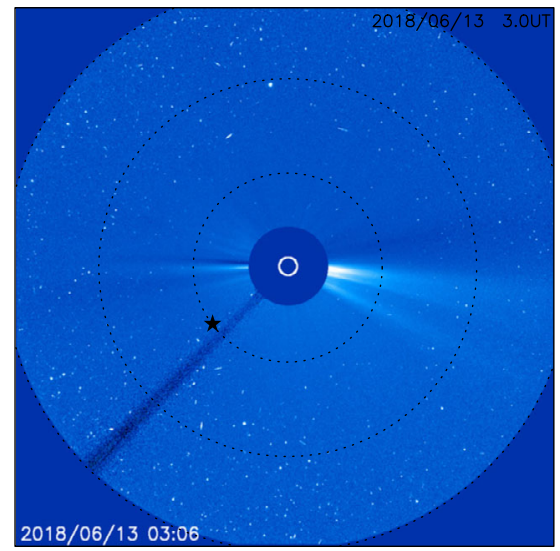

(c)

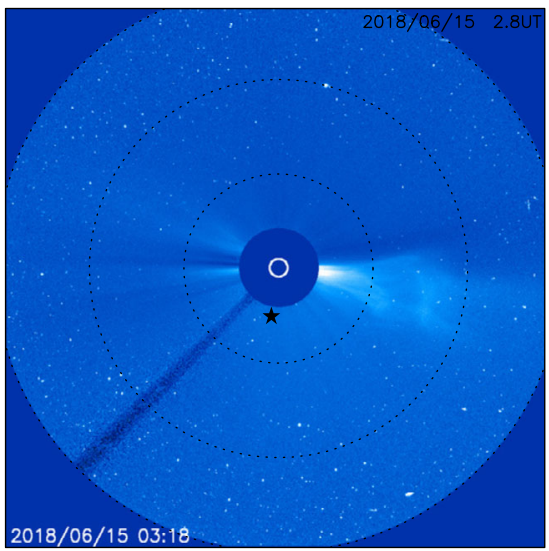

(e)

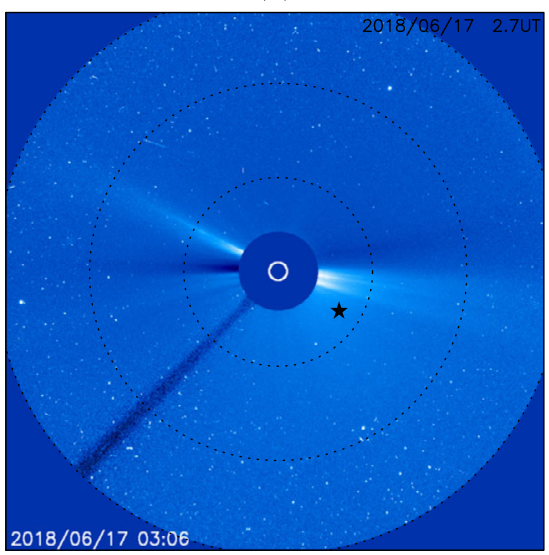

(b)

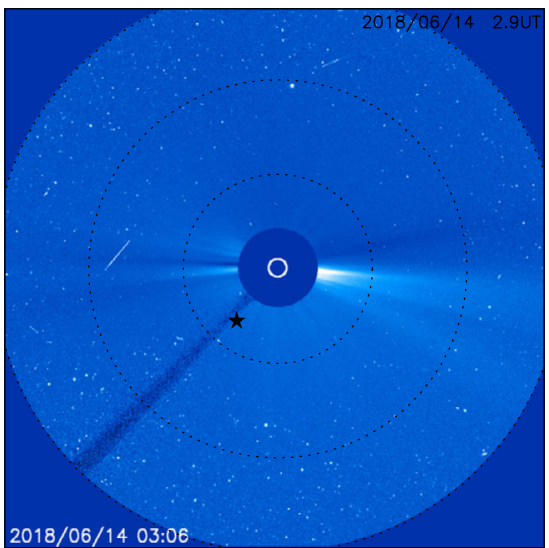

(d)

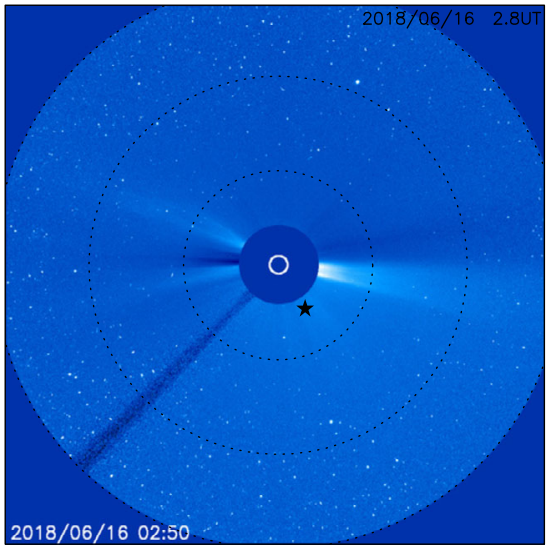

(f)

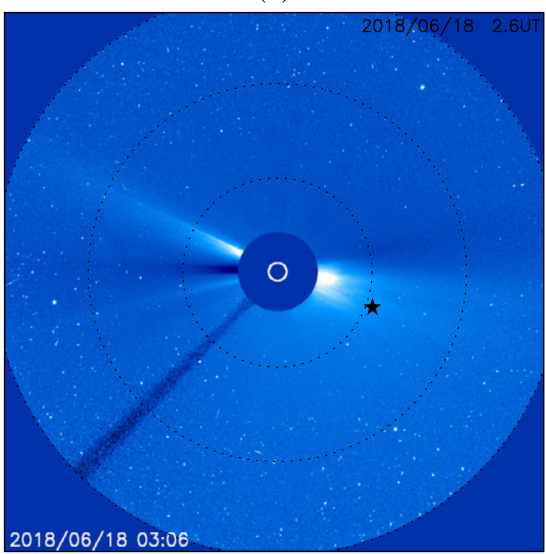

Figure 11 LASCO C3 images for (a-f) 13-18 June 2018. The observation times for LASCO C3 and Crab pulsar are indicated at the bottom left and top right of each panel, respectively. The location of the LOS for Crab pulsar is indicated by the black star. 
(a)

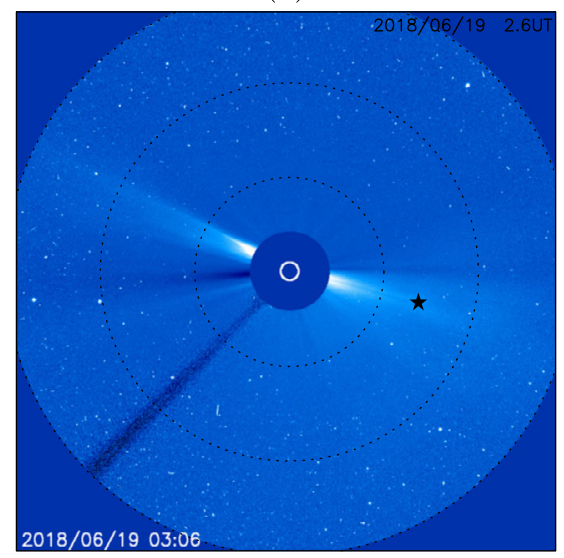

(c)

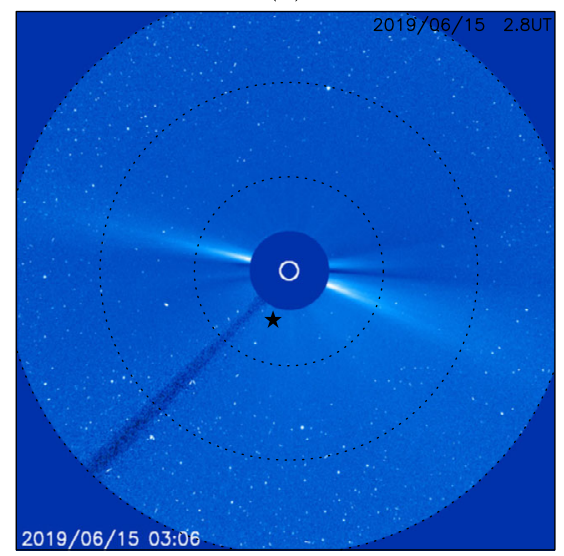

(e)

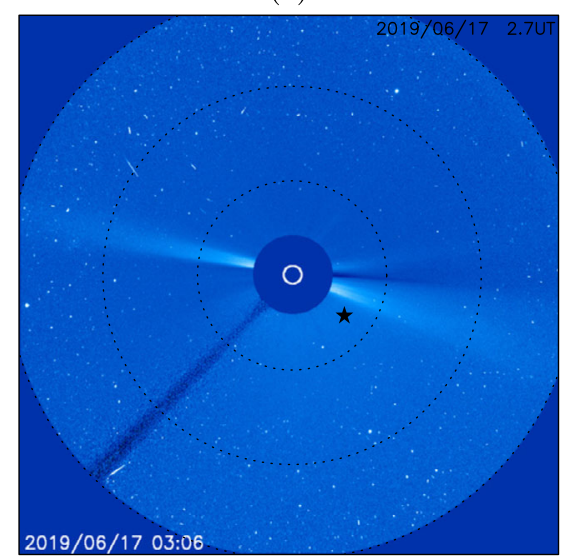

(b)

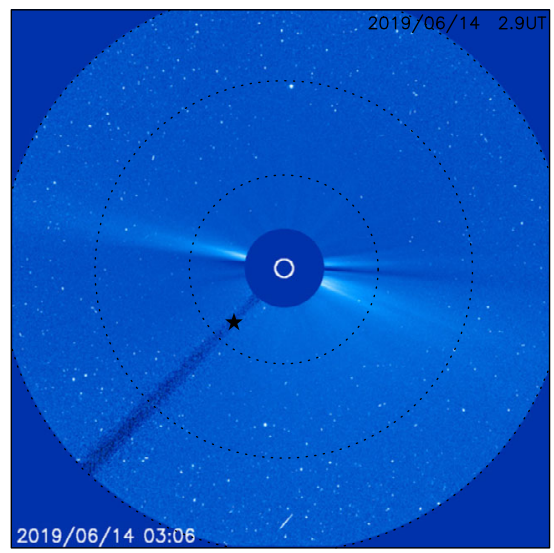

(d)

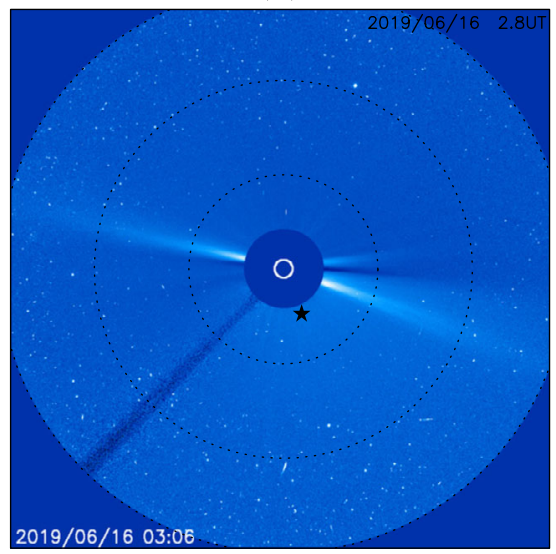

(f)

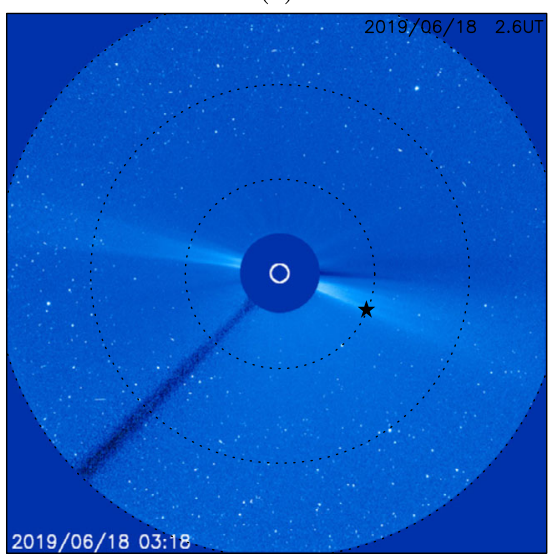

Figure 12 LASCO C3 images for (a) 19 June 2018 and (b-f) 14-18 June 2019. The observation times for LASCO C3 and Crab pulsar are indicated at the bottom left and top right of each panel, respectively. The location of the LOS for Crab pulsar is indicated by the black star. 
away from it (see Figure 11c). No CME was identified from the LASCO C3 images at the beginning and the end of the observation periods. Therefore, the effect of CMEs can be safely ignored in this study.

\section{Discussions}

A significant drop in the solar wind mass and momentum flux was revealed from in situ observations in the early phase of SC24, whose activity has been the weakest over the past 100 years. The mass flux and dynamic pressure of the solar wind observed for 2009-2013 decreased by $34 \%$ and $41 \%$, respectively, compared to those for 1974-1994 (McComas et al., 2013). Furthermore, the solar wind speed reduced insignificantly (by 11\%), and therefore the change in mass flux and dynamic pressure mostly comprised a reduction in the density (by $27 \%$ ). A distinct long-term decline in the solar wind density fluctuations $\left(\Delta N_{e}\right)$ was reported from remote sensing observations using interplanetary scintillation (IPS) (Janardhan et al., 2011, 2015; Bisoi et al., 2014; Sasikumar Raja et al., 2019). $\Delta N_{e}$ is considered as a proxy of the solar wind density, and IPS observations are consistent with the in situ ones. These observations suggest that a drastic change in the plasma density distribution near the Sun may occur in SC24. Contrary to this expectation, the results obtained here demonstrate that the derived plasma density distribution near the Sun was almost the same as that observed in SC20 and 22 declining phases (models a and b). A possible explanation for this discrepancy is that the coronal plasma condition in 2018-2019 recovered to that in the low solar activity period of past cycles. According to the in-ecliptic in situ measurements at 1 $\mathrm{AU}$, the solar wind dynamic pressure abruptly increased in late 2014 and gradually declined from 2017 to 2019 while maintaining the level observed in the previous solar minimum (McComas et al., 2020). This is consistent with the results obtained in this study. In contrast to the in situ data, solar microwave observations conducted continuously from 1957 to 2016 demonstrated that the microwave spectra at five solar minima between SC19/20 and SC23/24 agree with each other, which suggests that the atmospheric structure above the upper chromosphere in the quiet Sun did not vary for half a century (Shimojo et al., 2017). If so, the reduction in the solar wind density for SC24 may have occurred at farther distances, beyond which the microwave observations were conducted.

The number of GPs observed at Toyokawa decreased when the LOS of the Crab pulsar was near the Sun. Since this decrease occurred in two occultation periods of different years repeatedly, it is unlikely that it has been caused by the intrinsic variations of GPs or the effect of the interstellar medium. We calculated the ray path for the $327 \mathrm{MHz}$ radio waves near the Sun and found that the effect of ray path bending was too small to account for the observed decrease in the GP occurrence. Furthermore, we measured the scattering timescale for observed GPs and found that the effect of pulse broadening cannot account for the decrease in the GP occurrence because there was no significant correlation between the measured scattering timescales and the GP occurrence during the closest approach to the Sun. Therefore, we consider that this decrease is caused by an increase in the background noise level through the sidelobes of SWIFT. The solar radio emission at $327 \mathrm{MHz}$ is very strong compared to the emission from the Crab pulsar, even when the Sun is in a quiet condition. Therefore, the solar radio missions detected by the sidelobes may raise the noise level or distort the beam pattern significantly, which can result in the degradation of the signal-to-noise ratios of GPs. The adaptive beamforming technique is useful for reducing the effect of solar radio emissions for Crab pulsar observations during the occultation period; however, this is beyond the scope of this study. 


\section{Summary}

We analyzed Crab pulsar observations made at Toyokawa between June 2018 and March 2020 to investigate the plasma density distribution near the Sun at the SC24/25 minimum. We derived DMs for intense GPs of the Crab pulsar and detected the increases in DM caused by the effect of coronal plasma during the closest approach of the LOS to the Sun in midJune 2018 and 2019. Further, we fit a spherically symmetric model (Equation 2) to the $D M$ data derived for GPs with $\mathrm{S} / \mathrm{N}>10$ and $\mathrm{S} / \mathrm{N}>15$. The DM data of GPs with $\mathrm{S} / \mathrm{N}>10$ yielded the best-fit models with smaller estimation errors. From the DM data of GPs with $\mathrm{S} / \mathrm{N}>10$, we obtained $N_{0}=6.37 \pm 8.82 \times 10^{3} \mathrm{~cm}^{-3}$ and $\alpha=1.50 \pm 0.98$ for 2018 and $N_{0}=7.30 \pm 2.69 \times 10^{3} \mathrm{~cm}^{-3}$ and $\alpha=1.33 \pm 0.15$ for 2019. In addition, we obtained $N_{0}=$ $6.49 \pm 4.46 \times 10^{3} \mathrm{~cm}^{-3}$ and $\alpha=1.37 \pm 0.32$ from the combined DM data of $\mathrm{S} / \mathrm{N}>10$ GPs for $2018-2019$. These models are similar and generally consistent with those obtained in Paper I. The estimation errors of the present study are considerably smaller than those of Paper I, and this is partly due to the better statistics with $\mathrm{S} / \mathrm{N}>10$ data and partly due to the careful assessment of the interstellar contributions. The radial slopes of the best-fit models obtained here are flatter than -2 corresponding to a uniform radial expansion. This is ascribed to the effect of the coronal hole over the South Pole. Supporting evidence for this interpretation is provided by comparing our DM data and LASCO C3 observations. The values of $N_{0}$ and $\alpha$ obtained here are similar to those obtained in earlier studies at the SC 20 and 22 declining phases. This suggests that the plasma density level at the SC24/25 minimum is almost the same as that in low-activity periods of the past cycle despite the significant reduction of the solar wind density in SC24. This suggests that the plasma density level near the Sun at SC24/25 minimum returned to that at low-activity periods of past cycles, which is consistent with the sudden recovery of the solar wind dynamic pressure after 2015 reported from in situ measurements.

Acknowledgments This study was performed under the Solar Wind Program of ISEE of Nagoya University. The DM data for the Crab pulsar at the Jodrell Bank Observatory were obtained from http://www.jb.man. ac.uk/ pulsar/crab.html. The synoptic maps of LASCO C3 observations were obtained from https://lascowww.nrl.navy.mil/carr_maps/. The LASCO C3 images were obtained from https://soho.nascom.nasa.gov/ data/. The authors would like to thank Drs. Kazumasa Iwai and Ken'ichi Fujiki (ISEE, Nagoya University) for their comments.

Data Availability Data used in the current study are available from the corresponding author on reasonable request.

\section{Declarations}

Disclosure of Potential Conflicts of Interest The authors declare that they have no conflicts of interest.

Open Access This article is licensed under a Creative Commons Attribution 4.0 International License, which permits use, sharing, adaptation, distribution and reproduction in any medium or format, as long as you give appropriate credit to the original author(s) and the source, provide a link to the Creative Commons licence, and indicate if changes were made. The images or other third party material in this article are included in the article's Creative Commons licence, unless indicated otherwise in a credit line to the material. If material is not included in the article's Creative Commons licence and your intended use is not permitted by statutory regulation or exceeds the permitted use, you will need to obtain permission directly from the copyright holder. To view a copy of this licence, visit http://creativecommons.org/licenses/by/4.0/.

\section{References}

Allen, C.W.: 1947, Mon. Not. Roy. Astron. Soc. 107, 426. DOI. 
Bird, M.K., Volland, H., Paetzold, M., Edenhofer, P., Asmar, S.W., Brenkle, J.P.: 1994, Astrophys. J. 426, 373. DOI.

Bisoi, S.K., Janardhan, P., Ingale, M., Subramanian, P., Ananthakrishnan, S., Tokumaru, M., et al.: 2014, Astrophys. J. 795, 69. DOI.

Brueckner, G.E., Howard, R.A., Koomen, M.J., Korendyke, C.M., Michels, D.J., Moses, J.D., et al.: 1995, Solar Phys. 162, 357. DOI.

Cognard, I., Bourgois, G., Lestrade, J.-F., Biraud, F., Aubry, D., Darchy, B., et al.: 1996, Astron. Astrophys. 311, 179.

Counselman, C.C., Rankin, J.M.: 1972, Astrophys. J. 175, 843. DOI.

Counselman, C.C., Rankin, J.M.: 1973, Astrophys. J. 185, 357. DOI.

Edenhofer, P., Esposito, P.B., Hansen, R.T., Hansen, S.F., Lüneburg, E., Martin, W.L., et al.: 1977, J. Geophys. 42, 673 .

Esposito, P.B., Edenhofer, P., Lueneburg, E.: 1980, J. Geophys. Res. 85, 3414. DOI.

Fox, N.J., Velli, M.C., Bale, S.D., Decker, R., Driesman, A., Howard, R.A., et al.: 2016, Space Sci. Rev. 204, 7. DOI.

Heiles, C., Campbell, D.B.: 1970, Nature 226, 529. DOI.

Janardhan, P., Bisoi, S.K., Ananthakrishnan, S., Tokumaru, M., Fujiki, K.: 2011, Geophys. Res. Lett. 38, L20108. DOI.

Janardhan, P., Bisoi, S.K., Ananthakrishnan, S., Tokumaru, M., Fujiki, K., Jose, L., et al.: 2015, J. Geophys. Res. 120, 5306. DOI.

Leblanc, Y., Dulk, G.A., Bougeret, J.-L.: 1998, Solar Phys. 183, 165. DOI.

Lyne, A.G., Pritchard, R.S., Graham Smith, F.: 1993, Mon. Not. Roy. Astron. Soc. 265, 1003. DOI.

Mancuso, S., Garzelli, M.V.: 2013, Astron. Astrophys. 560, L1. DOI.

McComas, D.J., Ebert, R.W., Elliott, H.A., Goldstein, B.E., Gosling, J.T., Schwadron, N.A., et al.: 2008, Geophys. Res. Lett. 35, L18103. DOI.

McComas, D.J., Angold, N., Elliott, H.A., Livadiotis, G., Schwadron, N.A., Skoug, R.M., et al.: 2013, Astrophys. J. 779, 2. DOI.

McComas, D.J., Bzowski, M., Dayeh, M.A., DeMajistre, R., Funsten, H.O., Janzen, P.H., et al.: 2020, Astron. Astrophys. Suppl. Ser. 248, 26. DOI.

Mercier, C., Chambe, G.: 2015, Astron. Astrophys. 583, A101. DOI.

Muhleman, D.O., Anderson, J.D.: 1981, Astrophys. J. 247, 1093. DOI.

Muhleman, D.O., Esposito, P.B., Anderson, J.D.: 1977, Astrophys. J. 211, 943. DOI.

Müller, D., St. Cyr, O.C., Zouganelis, I., Gilbert, H.R., Marsden, R., Nieves-Chinchilla, T., et al.: 2020, Astron. Astrophys. 642, A1. DOI.

Newkirk, G.: 1961, Astrophys. J. 133, 983. DOI.

Saito, K., Poland, A.I., Munro, R.H.: 1977, Solar Phys. 55, 121. DOI.

Sasikumar Raja, K., Janardhan, P., Bisoi, S.K., Ingale, M., Subramanian, P., Fujiki, K., et al.: 2019, Solar Phys. 294, 123. DOI.

Shimojo, M., Iwai, K., Asai, A., Nozawa, S., Minamidani, T., Saito, M.: 2017, Astrophys. J. 848, 62. DOI.

Smirnova, T.V., Chashei, I.V., Shishov, V.I.: 2009, Astron. Rep. 53, 252. DOI.

Staelin, D.H.: 1970, Nature 226, 69. DOI.

Staelin, D.H., Reifenstein, E.C.: 1968, Science 162, 1481. DOI.

Stelzried, C.T., Levy, G.S., Sato, T., Rusch, W.V.T., Ohlson, J.E., Schatten, K.H., et al.: 1970, Solar Phys. 14, 440. DOI.

Tiburzi, C., Shaifullah, G.M., Bassa, C.G., Zucca, P., Verbiest, J.P.W., Porayko, N.K., et al.: 2021, Astron. Astrophys. 647, A84. DOI.

Tokumaru, M., Kojima, M., Fujiki, K., Maruyama, K., Maruyama, Y., Ito, H., et al.: 2011, Radio Sci. 46, RS0F02. DOI.

Tokumaru, M., Tawara, K., Takefuji, K., Sekido, M., Terasawa, T.: 2020, Solar Phys. 295, 80. DOI.

Tyler, G.L., Brenkle, J.P., Komarek, T.A., Zygielbaum, A.I.: 1977, J. Geophys. Res. 82, 4335. DOI.

Weisberg, J.M., Rankin, J.M., Payne, R.R., Counselman, C.C.: 1976, Astrophys. J. 209, 252. DOI.

Wexler, D.B., Hollweg, J.V., Efimov, A.I., Song, P., Jensen, E.A., Lionello, R., et al.: 2019a, J. Geophys. Res. 124, 7761. DOI.

Wexler, D.B., Hollweg, J.V., Efimov, A.I., Lukanina, L.A., Coster, A.J., Vierinen, J., et al.: 2019b, Astrophys. J. 871, 202. DOI.

Publisher's Note Springer Nature remains neutral with regard to jurisdictional claims in published maps and institutional affiliations. 